\title{
Las buenas prácticas de gobierno corporativo en empresas familiares del sector carrocero de Duitama, Colombia
}

\section{Good corporate governance practices in family businesses in the coach building sector of Duitama, Colombia}

\author{
María del Pilar Báez-Roa' \\ Gloria Acened Puentes-Montañez ${ }^{2}$ \\ Viviana Catalina Sosa ${ }^{3}$
}

\begin{abstract}
Recibido: junio 26 de 2020
Aceptado: noviembre 02 de 2020

\section{Resumen}

El objetivo de la investigación fue identificar las buenas prácticas de gobierno corporativo en empresas familiares de la industria carrocera, ubicadas en el departamento de Boyacá, Colombia. El estudio tuvo un enfoque cualitativo, con investigación descriptiva y diseño de campo no experimental, utilizando como técnica de recolección de información la entrevista semiestructurada. La muestra correspondió a 10 empresas de carácter familiar, donde se entrevistó a los propietarios de las mismas. Los hallazgos mostraron que la mayoría de las empresas se encuentran en la primera generación, y no han considerado la aplicación de manera formal de las buenas prácticas de gobierno corporativo. No obstante, se observa que hay presencia intuitiva de esas prácticas, aspecto que se refleja en: el bajo nivel de conflictos familiares, aprovechamiento de las ventajas de ser empresa familiar y aplicación de una gestión participativa, lo que ha llevado

Abstract

The objective of the research was to identify good corporate governance practices in family companies in the auto body industry, located in the department of Boyacá, Colombia. The study had a qualitative approach, with descriptive research and non-experimental field design, using the semi-structured interview as the information gathering technique. The sample corresponded to 10 family businesses, where their owners were interviewed. The findings showed that most companies are in the first generation, and have not considered the formal application of good corporate governance practices. However, it is observed that there is an intuitive presence of these practices, an aspect that is reflected in: the low level of family conflicts, taking advantage of the advantages of being a family business and applying participatory management, which has led to the sustainability of the long-term companies.
\end{abstract} a la sostenibilidad de las empresas a largo plazo.

Palabras clave: gobierno corporativo, empresa familiar, pequeñas y medianas empresas, teoría de la agencia.
Keywords: corporate governance, family business, small and medium businesses, agency theory.

1 Administradora Industrial, Magíster en Administración, Universidad Pedagógica y Tecnológica de Colombia, Duitama, Colombia. E-mail: mariadelpilar.baez@uptc.edu.co

ORCID: https://orcid.org/0000-0002-9123-7139

2 Administradora de Empresas Agropecuarias, Estudiante de Doctorado en Proyectos, Universidad Pedagógica y Tecnológica de Colombia, Duitama, Colombia. E-mail: gloria.puentes@uptc.edu.co ORCID: https://orcid.org/0000-0002-5953-9591

3 Profesional en Relaciones Internacionales y Estudios Políticos, Corprogreso, Bogotá, Colombia. E-mail: sosaviviana03@gmail.com ORCID: https://orcid.org/0000-0003-1377-688X 


\section{Introducción}

En Colombia, el sector carrocero es uno de los más dinámicos del país, como se evidencia en la Encuesta Anual Manufacturera, realizada por el Departamento Administrativo Nacional de Estadística (2018), donde los vehículos automotores, remolques y semirremolques tuvieron una variación positiva de 9,2\%. Asimismo, en la Encuesta Mensual Manufacturera para agosto, se indica que el sector con los mejores resultados fue el de fabricación de carrocerías para vehículos automotores y remolques, que tuvo un incremento del 38,9\%, con relación al mismo mes de 2017, a la vez que sus ventas se impulsaron $19,2 \%$ y el empleo un $14,5 \%$, ( $\mathrm{La}$ República, 2018). Igualmente, en la Agenda Integrada de Competitividad, Ciencia, Tecnología e Innovación del departamento de Boyacá, se reporta que la industria Siderúrgica aporta el 40 $\%$ de la producción nacional (Comisión Regional de Competitividad de Boyacá, 2019). Por ello, Boyacá se considera como un departamento importante en esta industria, fortalecida por la presencia de empresas ensambladoras de vehículos de carga y pasajeros.

Las empresas del sector carrocero de Boyacá, en su gran mayoría, son empresas familiares, las cuales datan de los años 60 . Inicialmente, la fabricación fue de forma artesanal, lo que incentivó el empleo de personal poco calificado, que aprendía su oficio en el taller. Esta característica, hizo que muchos trabajadores tomaran la decisión de crear su propio taller, como lo comentan los que son hoy en día grandes empresarios del sector. Otro factor que impulsó el desarrollo de esta industria, fue la creación de la Ciudadela Parque Industrial de Duitama, donde se ubican varias empresas carroceras y otros empresarios de la región, lo que permitió moldear el clúster metalmecánico carrocero de Duitama (Portafolio, 2018).

Lo anterior podría obedecer a modelos que explican la influencia que tienen factores del entorno, la formación y la experiencia en las competencias personales, y estas en las intenciones emprendedoras, que finalmente llevan a la creación de la empresa, (Reilly \& Carsrud, 2000, citados por Bernadich, 2013). La creación de las empresas familiares, tiene como objetivo el forjar un patrimonio propio sin depender de terceras personas, y trabajar unidos generando empleo para ellos mismos (González-Macías \& Sánchez-Juárez, 2017).

Según Tagiuri y Davis (1996), las empresas familiares pueden tener tres variantes, a saber: cuando la empresa está controlada por sus propietarios (propiedad), cuando la empresa está administrada por al menos dos miembros de la familia (familia) y cuando en la administración hay empleados que no son familiares (empresa). Estos aspectos crean superposiciones, que hacen que las empresas de familia tengan sus propias características, pudiendo ser una ventaja o desventaja para la empresa. Como desventaja, se tiene que dichas superposiciones traen conflictos que es necesario resolver, mediante la implementación de estructuras de gobierno corporativo familiar. Estas estructuras tienen por finalidad el definir claramente las funciones de cada rol, determinar el límite entre los asuntos de la familia y la empresa, entre otras, lo que a su vez busca garantizar la sostenibilidad de la empresa a largo plazo.

La presente investigación busca identificar las buenas prácticas de gobierno corporativo, en la estructura administrativa de las empresas familiares del sector carrocero de Duitama, departamento de Boyacá. En este sentido, se aportará a la comprensión de las relaciones de propiedad, familia y administración de las empresas carroceras, lo cual servirá como referente para direccionar las prácticas en su gobernanza. A continuación se presenta el marco teórico, iniciando con la definición del concepto de empresa familiar para el contexto empresarial. Luego se expone el modelo familiar y la dinámica de la empresa familiar, continuando con la metodología del estudio, junto con los resultados y las conclusiones de la investigación. 


\section{Marco teórico y metodología}

\subsection{Empresas familiares}

Las definiciones de empresa o negocio familiar no tienen un consenso dentro de la comunidad académica, esto a causa de su variedad, ya que no existen dos empresas familiares idénticas. En este sentido, se encuentran empresas pequeñas y grandes, fundadoras y multigeneracionales, entre otras. La variedad también se da por la intersección entre dos sistemas, el familiar o empresarial (Neubauer \& Lank, 1998); o de tres sistemas: familiar, propiedad y empresarial, más conocido como el modelo de los tres círculos (Tagiuri \& Davis, 1996).

La diversidad en las empresas familiares lleva a mayores conflictos entre los temas familiares y empresariales, tales como: concentración del poder en el fundador o accionista controlante, la ausencia de preparación para el cambio generacional, la carencia de idoneidad y liderazgo de los administradores, junto con la ausencia de procedimientos formales (Lara-Cogollo, 2009; Mojica, 2014). En una encuesta realizada por PricewaterhouseCoopers (2016), a más de 2.802 empresas de familia, de 50 países, se encontró que el $43 \%$ de estas empresas no tienen un plan de sucesión discutido y documentado. Asimismo, en el estudio de Beltrán (2006), se encontró que los problemas más frecuentes en las pymes, son: conflictos familiares en lo referente a reparto de utilidades, vinculación laboral y llegada de parientes políticos, entre otros.

La teoría de agencia hace referencia a la separación de la propiedad y el control, en donde el principal (Gerente actual), sede el poder de toma de decisiones en su nombre al agente (Nuevo gerente). Esta situación puede con llevar a que el nuevo gerente no tome las mejores decisiones para la empresa sino para su propio beneficio, creando el llamado conflicto de intereses (Jensen \& Meckling, 1976). Las empresas familiares no escapan a estos problemas de agencia, ya que por su estructura de propiedad pueden presentar conflictos de interés, no precisamente en la dirección de la empresa sino a la hora de la sucesión. Esto ya que, al seleccionar a los herederos, se pueden sentir celosos entre ellos y la familia extendida se puede sentir menospreciada, por lo que el plan de sucesión se prefiere mantener en la confidencialidad. Además, los propietarios tienden a dar mayores privilegios y beneficios a los miembros familiares, (Schulze et al., 2001), acción que hace que los empleados no familiares puedan estar insatisfechos y menos motivados ya que sus recompensas son menores (Dyer, 1994).

Los conflictos de roles y el compromiso con múltiples grupos de interés, son problemas comunes para las empresas familiares. "Un miembro de familia trabajando en una empresa familiar puede ocupar diferentes roles simultáneamente, como, por ejemplo: padre, hijo, hermano, un empleado o un propietario; a menudo es difícil para un miembro de la familia, que desempeña roles importantes, determinar qué rol es apropiado en una situación dada" (Tagiuri \& Davis, 1996). En este sentido, se han desarrollado herramientas para mitigar los conflictos, como son las buenas prácticas de gobierno corporativo, las cuales buscan: "facilitar la creación de un ambiente de confianza, transparencia y rendición de cuentas, necesario para: favorecer las inversiones a largo plazo, la estabilidad financiera y la integridad en los negocios" (OCDE, 2006).

\subsection{Estructuras de gobierno corporativo familiar}

Las empresas familiares, al igual que las no familiares, necesitan definir estructuras de gobierno que ofrezcan lineamientos para una convivencia en armonía. Sarbah \& Xiao (2015), afirman que contar con un sistema de gobierno ayuda a resolver conflictos, permitiendo que la familia propietaria se concentre en el negocio. Así mismo, Mancilla-Rendón y Saavedra-García (2015), se refieren al gobierno corporativo, como: los métodos que se llevan a cabo en las empresas para establecer normas, que permitan la buena toma de decisiones, y se vean representados los intereses colectivos. La International Finance 
Corporation, IFC (2018), en su Manual de gobierno corporativo para sociedades cerradas, propone la implementación de: la asamblea de familia, consejo de familia y el protocolo de familia, cuyas funciones se describen a continuación.

Tabla 1. Asamblea de familia.

\begin{tabular}{ll}
\hline \multicolumn{1}{c}{ Autores } & \multicolumn{1}{c}{ Funciones } \\
\hline CAF (2006) & Es de carácter informativo. \\
& Es consultiva. \\
& Conformada por toda la familia. \\
& Fomenta el intercambio de opiniones. \\
& Consultivo, informativo. \\
Superintendencia de & Comunicación en un ambiente de confianza. \\
Sociedades et al. (2009) & Trmonía familiar, Inculcar valores. \\
& Podos tienen derecho a voto. \\
& miembros más jóvenes de la familia. \\
& Velar por el cumplimiento del protocolo de familia. \\
& Nombrar los integrantes del consejo de familia. \\
IFC (2018) & Intercambio de ideas. \\
& Educación de los miembros de la familia. \\
& Elección de los miembros del consejo de familia. \\
& Aprobación del protocolo de familia \\
\hline
\end{tabular}

En la Tabla 1 se puede identificar como principal indicador de la Asamblea de familia, las Reunio- nes para intercambiar ideas, inculcar valores y compartir.

Tabla 2. Protocolo de familia.

\begin{tabular}{ll}
\hline \multicolumn{1}{c}{ Autor } & \multicolumn{1}{c}{ Funciones } \\
\hline CAF (2006) & Definir la cultura y los valores familiares, haciendo \\
& explícitas su misión y visión, su historia. \\
& Definición de planes de sucesión. \\
& Regular las funciones de las asambleas y los consejos de familia. \\
& Clarificar las normas de incorporación de los \\
& miembros de la familia a la empresa. \\
& Limitar el involucramiento de familiares y allegados políticos. \\
& Normas claras en relación con el establecimiento de negocios \\
& que puedan competir con la empresa familiar. \\
& Determinar los límites máximos de propiedad \\
& para accionistas diferentes a la familia. \\
& Historia de la familia, de la empresa, los valores que guían sus actuaciones. \\
& La profesionalización del negocio. \\
& Las normas que regulan las relaciones laborales, \\
Superintendencia de & y otro tipo de relaciones económicas. \\
Deciedades et al. (2009) & Regular los órganos de gobierno: asamblea y consejo familiar. \\
& Procedimiento para la solución de controversias \\
entre los diferentes miembros de la familia.
\end{tabular}


Compromiso de la familia con los valores centrales, la visión y la misión de la empresa.

Definición de los roles, composiciones y poderes de los principales órganos de gobierno.

Definición de las relaciones entre los órganos de gobierno y cómo los miembros de la familia pueden participar de manera significativa en el gobierno.

En la Tabla 2 se identifican como principales indicadores de Protocolo de familia: Valores que guían sus acciones; Normas de vinculación para la familia propietaria, no propietario y familiar político; Manejo de los conflictos para la familia propietaria, no propietario, familiar político; y plan de sucesión empresarial.

Tabla 3. Consejo de familia.

\begin{tabular}{|c|c|}
\hline Autor & Funciones \\
\hline CAF (2006) & $\begin{array}{l}\text { Decisiones sobre las relaciones empresa y familia. } \\
\text { Es el órgano que aplica las normas y } \\
\text { contenidos de la empresa familiar. }\end{array}$ \\
\hline Lansberg (1988) & $\begin{array}{l}\text { Asesor. } \\
\text { Articula la visión familiar para que los miembros en } \\
\text { la junta directiva tomen decisiones que protejan los } \\
\text { valores, necesidades y deseos de la familia. } \\
\text { Discutir diferencias respecto de la empresa sin intervenir en la } \\
\text { administración de la misma y sin afectar las relaciones familiares. }\end{array}$ \\
\hline $\begin{array}{l}\text { Superintendencia de } \\
\text { Sociedades et al. (2009) }\end{array}$ & $\begin{array}{l}\text { Se conforma por familiares propietarios. } \\
\text { Sirve como órgano consultivo para la Junta } \\
\text { Directiva de la sociedad de familia. } \\
\text { Decidir los asuntos que hacen referencia a las relaciones de } \\
\text { los miembros de la familia con la sociedad de familia. } \\
\text { Elegir a los representantes de la familia que serán } \\
\text { candidatos a ocupar cargos dentro de los órganos } \\
\text { de gobierno de la sociedad de familia. } \\
\text { Suscribir el Protocolo de Familia, } \\
\text { Velar por la divulgación y el cumplimiento } \\
\text { del Protocolo de Familia. }\end{array}$ \\
\hline IFC (2018) & $\begin{array}{l}\text { Ser el vínculo principal entre la familia, la junta y la alta gerencia. } \\
\text { Sugerir y discutir nombres de candidatos } \\
\text { para la membresía de la junta. } \\
\text { Redactar y revisar documentos de posición } \\
\text { familiar sobre su visión, misión y valores. } \\
\text { Redactar y revisar las políticas familiares, como: el empleo } \\
\text { familiar, compensación y participación familiar. }\end{array}$ \\
\hline
\end{tabular}

En la Tabla 3 se establecen como principales indicadores del Consejo de Familia: Estudiar situaciones y tomar decisiones acerca de la empresa; decidir las relaciones de los miembros de la familia con la empresa (Presencia de conflictos entre familiares propietarios, no propietarios y familiares políticos); servir como órgano consultivo para el Gerente (Junta directiva). 


\subsection{Metodología}

La investigación es cualitativa, ya que se observan fenómenos de la existencia de prácticas de gobierno corporativo en las mipymes carroceras, tal como se dan en su contexto natural, para posteriormente analizarlos (Hernández-Sampieri, Fernández-Collado \& Baptista-Lucio, 2010). Se trata de un estudio de caso con alcance descriptivo- interpretativo, para obtener un mayor entendimiento del fenómeno en estudio.

La población se estableció en la base de datos de la Cámara de Comercio de Duitama para 2019: empresas pertenecientes al código CIIU 2920, “Fabricación de carrocerías para vehículos automotores; fabricación de remolques y semirremolques". En ella se identificaron 13 empresas de conformación familiar. La muestra estuvo conformada por 10 empresas, que voluntariamente participaron en la investigación.

Las dimensiones de la estructura de gobierno seleccionadas para su análisis, fueron: asamblea familiar, consejo de familia y protocolo de familia. La información se recopiló a través de una entrevista semiestructurada, aplicada a los propietarios de las empresas participantes. El instrumento se evaluó por expertos y se realizó una prueba piloto, a partir de la cual se hicieron algunos ajustes.
Las etapas para el análisis e interpretación de la información, se basaron en Díaz et. Al. (2013), así: en la primera etapa se realiza la transcripción de la entrevista. Luego, en la etapa de teorización, la información se organiza en matrices de acuerdo a los ejes temáticos de la entrevista; se busca contrastar, realizar comparaciones, clasificar, ordenar definiendo categorías y sus propiedades, y establecer las correspondientes relaciones. Finalmente, se reflexiona sobre la información recolectada, con base en la triangulación, integrando teorías, documentos y testimonios, utilizando: matrices, tablas de frecuencia y el software para el análisis de la información.

\section{Resultados y discusión}

\subsection{Asamblea de familia}

En la tabla 4, se presentan las características de las empresas estudiadas, todas ellas de origen familiar. Se observa que la más antigua tiene 42 años de fundada, mientras que la más reciente tiene tan solo 10 años. Además, 6 de las 10 empresas están administradas por los fundadores, mientras que las 4 restantes se encuentran en la segunda generación.

Tabla 4. Empresas estudiadas.

\begin{tabular}{cllllll}
\hline Empresa & Tamaño & Propietarios & Gerente & Generación & $\begin{array}{c}\text { Fecha de } \\
\text { creación }\end{array}$ & $\begin{array}{c}\text { Familiares } \\
\text { trabajadores }\end{array}$ \\
\hline 1 & Pequeña & Fundador & Fundador & Primera & 1993 & la esposa \\
2 & Micro & Fundador & Fundador & Primera & 1996 & Hermano \\
3 & Micro & Mamá y 4 hijos & Hijo & Segunda & 1980 & una hija \\
4 & Pequeña & Fundador & Fundador & Primera & 1998 & No \\
5 & Pequeña & mamá y dos hijos & Hijo & Segunda & 2010 & No \\
6 & Pequeña & $\begin{array}{l}\text { Mamá y } \\
\text { cinco hijos }\end{array}$ & Fundador & Primera & 2006 & 3 hermanos \\
8 & Pequeña & mamá e hijo & Fundadores & Primera & 2007 & esposa del dueño \\
9 & Pequeña & Esposos & Hermano & Segunda & 1998 & 4 hermanos \\
& & Papá & Primera & 1992 & 6 entre Hijo, tío \\
10 & Mediana & $\begin{array}{l}50 \% \text { col hijo y 25\% } \\
\text { cada hermana }\end{array}$ & Hijo & Segunda & 1980 & No hay \\
\hline
\end{tabular}


En cuanto a la asamblea de familia, esta genera espacios de intercambio (reuniones), donde la familia propietaria interactúa en un ambiente de armonía. Esto hace que se den diálogos informales acerca de lo que está sucediendo con la familia y la empresa, donde se pide consejo a los mayores, permitiendo una mayor comprensión y conciliación, lo que lleva a afianzar la confianza y los lazos familiares. En este caso, las relaciones intergeneracionales se reflejan en las siguientes apreciaciones:

"Normalmente estamos reuniéndonos como familia, constantemente cuando hay negocios nuevos se pide consejo a los mayores, papá y mamá; ellos dicen si conviene o no, hágalo o no lo haga". (Empresa 5).

"muchas veces me reúno con mis papás para que me den consejos respecto a lo que está sucediendo, especialmente mi papá". (Empresa 1).

Lo anterior es consistente con Folle (2016), quien encontró que la confianza fomenta la unidad y conlleva a la felicidad de todos los miembros de la familia; esto va unido a efectos económicos positivos, ya que disminuye los costos de transacción, que están unidos a utilizar mecanismos de control complejos. En otra investigación sobre 285 empresas familiares de Colombia y México, se evidenció que "la probabilidad de tener una percepción de mejoramiento en la unidad y armonía es más alta -2.39 veces-, cuando la persona que lidera el patrimonio considera necesario el consejo de otros para el buen desarrollo de la empresa", (Gómez-Betancourt et al., 2016).

\subsection{Protocolo de familia}

En las empresas familiares convergen dos aspectos: familia y empresa, los cuales generan superposición de roles que usualmente crean conflictos, lo que a su vez afecta la toma objetiva de decisiones. En este sentido, es necesario definir un Protocolo de familia, de manera voluntaria, que regule los diferentes roles y relaciones entre la familia y la empresa. En las mipymes carroceras de Duitama, se encontraron los siguientes lineamientos:

\section{Valores}

Estos se reflejan en aspectos como: comunicación, trabajo en equipo, equidad, respeto, solidaridad y compromiso (Salas-Arbeláez, García-Solarte \& Azuero-Rodríguez, 2018). Los valores definen el comportamiento de las empresas familiares, como se observó en la misión, visión y los objetivos de las empresas estudiadas. Lo anterior es consistente con Arslan y Alqatan (2020), quienes afirman que en las instituciones se forman las restricciones sociales y se hace referencia a las políticas, así como las restricciones no formales que tienen que ver con la cultura, en donde la familia es considerada como la fuente de identidad, con un vínculo fuerte, con responsabilidad y lealtad; estos aspectos salvaguardan las tradiciones, las costumbres y la religión.

El mito del fundador, en este caso, está dado por aquellos valores que él desarrolla y a la vez inculca a sus familiares. En la investigación de Coutinho-Lourenço de Lima, Vieira y Honório (2015), sobre tres empresas familiares, se encontró que los valores destacados del fundador, son: humildad y dedicación, cooperación y colaboración.

\section{Normas de vinculación de la familia}

Propietarios. Las diez empresas realizan contratos de trabajo, y declaran las funciones con los familiares propietarios que trabajan en la empresa. Respecto al tipo de relación que se da en la empresa, la mayoría hizo las siguientes afirmaciones: hay una relación de jefe y empleado muy clara; no se mezcla lo personal con lo laboral.

Las normas, roles y responsabilidades claras entre los miembros de la familia propietaria, permiten una relación positiva, reduciendo los conflictos y dejándoles más tiempo para concentrarse en el negocio (Romero, 2006). Asimismo, Basco (2010), en un grupo de empresas familiares españolas, encontró que la profesionalización "está orientada a potenciar una coordinación óptima hacia los recursos y capacidades, basada en los objetivos que persigue". 
Familiares no propietarios. Solo dos empresas emplean a familiares no propietarios, los cuales cuentan con su contrato de trabajo y asignación de funciones. Respecto a la relación, un empresario comenta:

"El hijo dice: aquí en la empresa no tengo papá, tíos, ni primos, somos compañeros de trabajo y como tal nos tratamos. Se debe cumplir el horario y los permisos deben ser justificados". (Empresa 9).

Familia política. Solo tres empresas tienen a familiares políticos, con contrato de trabajo y asignación de funciones. Según Arslan y Alqa$\tan (2020)$, la mayoría de empresas familiares en Pakistán, nombran a los miembros de la familia en cargos directivos y no directivos, atendiendo a la incidencia de las costumbres, los valores y a la puesta en marcha de las buenas prácticas de gobierno corporativo. No obstante, los autores recomiendan trabajar con personal directivo independiente, que garantice la equidad en la toma de decisiones, así como la protección de derechos y activos a los accionistas minoritarios.

\section{Manejo de conflictos}

Entre familiares propietarios. De las 10 empresas, solo una ha tenido conflictos entre los propietarios. En general, se evidencia una relación armoniosa de cariño conciliación, por lo que los conflictos que surgen, se resuelven por medio del diálogo o no son importantes. En este sentido, "la mayoría de familias empresarias cuentan con una fortaleza intrínseca para resolver conflictos a lo largo de los años, perseverando frente a las dificultades y comprendiendo que a veces son necesarios esfuerzos para adaptarse bien a los cambios. Compartir vínculos emocionales ayuda a la cohesión natural y a participar en objetivos comunes" (Chiner, 2011).

Entre familiares no propietarios. De las $10 \mathrm{em}-$ presas, solo dos han tenido familiares no propietarios. Una de ellas decidió establecer como política la no contratación de familiares no propietarios, por la frecuencia de conflictos. La otra empresa, maneja los conflictos por medio de los comités de COPASST (Comité Paritario de Salud y Seguridad en el Trabajo), mediante el diálogo. Entre las apreciaciones, se tienen:

"Los problemas que se presentan afectan la gestión, ya que los mismos miembros de la familia toman una actitud diferente. En las actividades de cada uno de ellos no rinden de la misma manera, ya cuando solucionan el problema y las diferencias, el consejo de familia opta por incentivarlos a cumplir con su trabajo y tomar la actitud adecuada para realizar sus actividades". (Empresa 9).

"En un momento adecuado, llamarlo hacerle las correcciones. Uno de ellos es muy terco y no entiende que la familia y la empresa no se mezclan y quiere tener más poder. Se han tenido conflictos con otros familiares, con los que se ha perdido la relación". (Empresa 9)

Se observa que la superposición de roles en las empresas familiares, son complejas de administrar ya que no se reconoce el rol apropiado para diferentes situaciones, llevando a diversos conflictos (Tagiuri \& Davis, 1996).

Con familiares políticos. Tres empresas tuvieron familiares políticos trabajando, con los cuales se presentaron conflictos y ya no se encuentran en la empresa. Esto se refleja en las siguientes afirmaciones:

"Hubo un cuñado que entró a reemplazarme en la parte gerencial, pero no fue posible que funcionara; posiblemente de pronto no funcionó porque él tenía criterios muy diferentes y quería que la empresa funcionara de una manera que no era conveniente". (Empresa 1)

En la empresa 7 también le dieron el cargo de administradora a la esposa del hijo, quién no supo dirigir la empresa y los estaba llevando a la quiebra, por lo que la mamá no permanecía en la empresa para evitar conflictos con la nuera.

"Una cuñada, no le gustaba la empresa, era muy desentendida, no llegaba los viernes, no venía, tenía algunas prebendas". (Empresa 10) 
En este caso, los conflictos que se presentaron con los parientes políticos fueron principalmente por la toma de decisiones erradas para la organización. Esto se atribuye a un problema de selección adversa, ya que los agentes no tienen las competencias y experiencia acordes al cargo, (Jensen \& Meckling, 1976). Para evitar estas situaciones, se puede aconsejar a ciertos líderes que no empleen a miembros de la familia. Esto elimina una fuente de conflictos para un líder que carece de la capacidad de gestionar las complejas relaciones trabajo-familia (Dyer, 1994).

\section{Plan de sucesión}

De las 10 empresas, cuatro pasaron a la segunda generación de una forma positiva, ya que los hijos permanecieron con los fundadores todo el tiempo, aprendiendo el oficio. Las seis empresas restantes no cuentan con un plan de sucesión. Esto tal vez porque la mayoría de las empresas son jóvenes, y no se vislumbra una persona para desarrollar un plan de sucesión. A este respecto, se tienen las siguientes afirmaciones:

"No veo a quien hacerle la sucesión, la empresa es muy joven". (Empresa 2)

"Se buscaría un comprador, o de pronto seguiría mi hermano". (Empresa 5)

En otra empresa, donde el hijo lleva varios años trabajando al lado del fundador, éste afirma: "el hijo dice yo estoy al lado de mi papá para seguir con la empresa, hacerla crecer; la idea es no dejar acabar lo que hizo mi papá" (Empresa 9).

Es así que los sucesores al estar trabajando en su empresa como primer empleo, viven un proceso de socialización, en el que se transmiten valores y se desarrolla un sentido de pertenencia dado por el compromiso y la satisfacción, lo cual los motiva a unirse a la empresa familiar en armonía (González, Guzmán, \& Trujillo, 2010; LozanoPosso \& Urbano, 2017).

Un hallazgo interesante es que dos microempresas, después de cerca de 10 años dirigidas por la segunda generación, actualmente se encuentran en crisis. Esto debido a la alta competencia que tiene el sector y a su baja profesionalización, entendida como la no adaptación al cambio. González-Macías y Sánchez-Juárez, (2017), indican que: "las empresas familiares ven comprometida su sostenibilidad y competitividad, debido a un enfoque tradicionalista de los propietarios-responsables, quienes no desean llevar a cabo las actividades de la empresa de una manera innovadora, al no aceptar la renovación ni el cambio".

\subsection{Consejo de familia}

Es necesario que el gerente reciba asesoría en cuestiones de la empresa por algunos propietarios, para que se protejan los intereses de la familia. En las empresas carroceras se encontró, de forma intuitiva, la constitución de consejos de familia que se reúnen cada vez que surge una situación importante de la empresa. Esto se refleja en las siguientes afirmaciones:

"Se reúne toda la familia empresaria cada que sea necesario para mostrar los logros: se evalúan las buenas prácticas de los trabajos, reorganizan los problemas, se visualiza lo que se hizo. se hace una reunión donde se caracteriza el problema y se le da un apoyo a lo que pueda cada cual". (Empresa 5)

"El papá se apoya siempre en mí en estos momentos, él me tiene en cuenta: hagamos esto o usted que le parece; yo le digo no me parece, mejor de esta forma, pero tiene que ser con credibilidad, con soluciones y tiempo". (Empresa 9)

En el trabajo de campo, se pudo constatar que las empresas carroceras de Duitama, cuando se trata de tomar decisiones relacionadas con la empresa, convocan a reuniones (consejos de familia). En ellos se da a conocer la información necesaria y se reciben los puntos de vista de cada uno, en un ambiente de colaboración y compañerismo que llevan a una toma de decisiones asertiva. En este sentido, Eddleston y Kellermanns (2007), afirman que: las empresas familiares con conflictos sufren de problemas 
en su desempeño, destacando los efectos positivos de un proceso de estrategia participativo. Los autores también encontraron que el altruismo puede ser un importante inhibidor de relaciones conflictivas, facilitando los procesos de estrategias participativas.

Algunas de las empresas estudiadas llevan un promedio de 20 años de existencia, por lo que todavía se encuentran en manos de sus fundadores. Es decir, el propietario es el mismo administrador, lo que permite la creación de un lazo de confianza lealtad, que beneficia las decisiones que se tomen en la empresa. De esta manera, los costos de agencia se eliminan entre el principal y el agente, ya que existen objetivos compatibles al ser la misma persona (Espinoza-Vásquez \& Rubin-de Celis Cedro, 2017).

\section{Conclusiones}

Las empresas carroceras de Duitama han logrado aprovechar las ventajas de ser una sociedad de familia y crear sinergias. Sobresale el hecho que en las empresas estudiadas, no se evidencian conflictos entre los familiares propietarios, lo que se podría atribuir a la característica principal de las relaciones familiares: el altruismo. Esto promueve que los padres cuiden a sus hijos, que los miembros de la familia se consideren entre ellos con mayor fraternidad, y que promuevan y sostengan el vínculo familiar.

Los resultados indican que los miembros de la familia propietaria que trabajan en la empresa, tienen claramente definidos sus roles y funciones, y son profesionales en su labor. Además, las funciones se cumplen a cabalidad y con responsabilidad, lo que limita la presencia de conflictos importantes. Otro factor relevante, son los valores que se han promovido en las familias, tales como: el trabajo en equipo, compromiso, la comunicación, el compañerismo y el respeto. En este sentido, se evidencian prácticas de buen gobierno, referentes al órgano de protocolo de familia, en cuanto a la regulación de las relaciones entre los propietarios.
En cuanto a los conflictos con familiares políticos, hubo un problema de selección adversa, lo que condujo a una crisis con altos costos de agencia. Esto debido a la falta de normas que regulen las extralimitaciones en las actuaciones de estos familiares. Respecto a los conflictos con empleados familiares no propietarios, estos se generaron por la percepción de estos individuos sobre preferencias en su labor, por ser familiares de los propietarios.

Otra característica de las mipymes carroceras de Duitama, es que la dirección de la empresa se encuentra en manos de los fundadores o de sus sucesores. Esto hace que se tengan objetivos compatibles en cuanto al bienestar de la familia, así como el interés por el rendimiento de la inversión, trabajando de una forma eficaz en la gestión de la empresa. Finalmente, el estudio permitió identificar algunos temas para futuras investigaciones, como: la gestión del aprendizaje de los propietarios y administradores de las empresas familiares, la cultura organizacional familiar en las empresas carroceras, y la gestión gerencial de las mipymes del sector carrocero.

\section{Referencias}

Arslan, M., \& Alqatan, A. (2020). Role of institutions in shaping corporate governance system: evidence from emerging economy. Heliyon, 6(13), 1-17. https://doi.org/https://doi.org/10.1016/j. heliyon.2020.e03520

Basco, R. (2010). Tipos de orientación familiar y prácticas de dirección y gobierno. Un estudio aplicado a las Empresas Familiares Españolas. Revista Europea de Dirección y Economía de la Empresa, 19 (2), 129-144.

Beltrán, A. (2006). Los 20 problemas de la pequeña y mediana empresa. Sotavento MBA, 7, 8-15.

Bernadich, D. (2013). Creación y desarrollo de empresas familiares: Una revisión de literatura desde el enfoque institucional. Nuevas investigaciones sobre la gestión de la empresa familiar en España, 11-44. https://doi.org/10.3926/oms.13 
Chiner, A. (2011). La necesidad de un buen gobierno de la familia en las empresas familiares. Universia Business Review, 32, 102-110.

Comisión Regional de Competitividad de Boyacá. (2019). Informe de la Agenda Integrada de Competitividad, Ciencia, Tecnología e Innovación del departamento de Boyacá. Tunja, Colombia.

Corporación Andina de Fomento, CAF. (2006). Manual de Gobierno Corporativo para empresas de capital cerrado. Recuperado de: http://www. iegc.ec/publicaciones/Manual_gc_empresas_ capital_cerrado CAF.pdf

Coutinho Lourenço de Lima, M., Vieira, A., \& Honório, L. (2015). a Construção Da Imagem Do Fundador Como Mito Na Empresa Familiar: Padrões Comportamentais E Relacionais. Gestão \& Planejamento, 16(3), 326-345.

Departamento Administrativo Nacional de Estadística. (2018). Boletín Técnico, encuesta anual manufacturera (EAM), 1-30.

Diaz, L., Torruco, U., Martínez, M., \& Varela, M. (2013). Uncertainty-based improved multidisciplinary design optimization methods. Investigación en educación médica, 2(7), 162-167. https:// doi.org/10.1109/IAEAC.2017.8054186

Dyer, G. (1994). Potential contributions of organizational behavior to the study of familyowned businesses. Family Business Review, 7(2), 109-131.

Eddleston, K., \& Kellermanns, F. (2007). Destructive and productive family relationships: $A$ stewardship theory perspective. Journal of Business Venturing, 22(4), 545-565. https://doi.org/ https://doi.org/10.1016/j.jbusvent.2006.06.004

Espinoza-Vásquez, L. M., \& Rubin-de Celis Cedro, R. (2017). Regulador no benevolente y asimetrías de información. Aproximación con un modelo de agencia. Revista de análisis económico, 32(1), 23-45. https://dx.doi.org/10.4067/S071888702017000100023

European Comission. (2015). Overview of family business: relevant issues. Recuperado de: http://
ec.europa.eu/DocsRoom/documents/10389/ attachment $/ 1 /$ translations

Folle, C. (2016). Confianza y poder en la empresa familiar ¿sustitutos o complementarios? IEEM Revista de Negocios, 19(5), 22-34.

Gómez-Betancourt, G., Betancourt-Ramírez, J., Lagos-Cortés, D., \& Aparicio-Castillo, R. (2016). Influencia de las prácticas de gobierno corporativo y gestión en la percepción de la unidad y armonía en empresas familiares. Revista Escuela De Administración De Negocios, (81), 159-174. https://doi.org/10.21158/01208160.n81.2016.1558

González, M., Guzmán, A., \& Trujillo, M. (2010). The role of heirs in family businesses: The case of Carvajal. Innovar, 20(36), 49-66.

González-Macías, C., \& Sánchez-Juárez, I. (2017). Sustentabilidad y competitividad en empresas familiares restauranteras. Revista Venezolana de Gerencia, 22(79), 427-446. https://doi.org/10.31876/ revista.v22i79.23032

Hernández-Sampieri, R., Fernández-Collado, C., \& Baptista-Lucio, P. (2010). Metodología de la Investigación. México: McGraw Hill.

International Finance Corporation. (2018). IFC Family Business Governance Handbook.

Jensen, M., \& Meckling, W. (1976). Racial diversity and its asymmetry within and across hierarchical levels: The effects on financial performance. Human Relations, 72(10), 1671-1696. https://doi. org/10.1177/0018726718812602

La República. (2018). Fabricación de carrocerías, el sector que más creció en agosto según el Dane. Recuperado de: https://www.larepublica. co/economia/fabricacion-de-carrocerias-elsector-que-mas-crecio-en-agosto-segun-eldane-2783096

Lansberg, I. (1988). The succession conspiracy, Family Busi-ness Review, 1(2), 119-143.

Lara-cogollo, M. (2009). Causas de cierre de empresas familiares en Colombia (Tesis de pregrado). Pontificia Universidad Javeriana. Bogotá. Recu- 
perado de: https://repository.javeriana.edu.co/ handle/10554/9040

Lozano-Posso, M., \& Urbano, D. (2017). Relevant factors in the process of socialization, involvement and belonging of descendants in family businesses. Innovar, 27(63), 61-76. https://doi. org/10.15446/innovar.v26n63.60667

Mancilla- Rendón, M., \& Saavedra-García, M. (2015). El gobierno corporativo y el comité de auditoría en el marco de la responsabilidad social empresarial. Contaduría y Administración, 60, 486-506.

Mojica, J. (2014). ¿Por qué la mayoría de empresas familiares no sobrevive a la tercera generación? Universidad Militar Nueva Granada.

Neubauer, F., \& Lank, A. (1998). The Family Businees: its Governance For sustainability. Mac Millan Press LTD. London: Hound Mills.

OCDE (2006). Principios de Gobierno Corporativo de la OCDE 2004. Madrid, España: Ministerio de Economía y Finanzas. https://doi. org/10.1787/9788485482726-es

Portafolio. (2018). El carrocero que le cambió la cara a la industria boyacense. Recuperado de: http://m.portafolio.co/negocios/el-carroceroque-le-cambio-la-cara-a-la-industria-boyacense-514931

PricewaterhouseCoopers PwC Family Business Survey, (2016). The 'missing middle':Bridging the strategy gap in family firms. Recuperado de: https://www.pwc.com/gx/en/family-businessservices/global-family-business-survey-2016/ pwc-global-family-business-survey-2016-themissing-middle.pdf

Romero, L. (2006). Competitividad y productividad en empresas familiares pymes. Revista Escuela de Administración de Negocios, (57), 131-141.

Salas-Arbeláez, L., García-Solarte, M., \& AzueroRodríguez, A. R. (2018). Género del gerente e innovación: Caso empírico en Pymes de Cali. Revista De Investigación, Desarrollo E Innovación,
8(2), 223-235. https://doi.org/10.19053/20278306. v8.n2.2018.7962

Sarbah, A., \& Xiao, W. (2015). Good Corporate Governance Structures: A Must for Family Businesses. Open Journal of Business and Management, 03(01), 40-57. https://doi.org/10.4236/ ojbm.2015.31005

Schulze, W., Lubatkin, M., Dino, R., \& Buchholtz, A. (2001). Agency relationships in family firms: theory and evidence, 12(2), 99-116. https://doi.org/ https://doi.org/10.1287/orsc.12.2.99.10114

Superintendencia de Sociedades, Cámara de Comercio de Bogotá, \& Confecámaras (2009). Guía Colombiana de gobierno corporativo para sociedades cerradas y de familia. Confecámaras. Recuperado de: http://www.confecamaras. org.co/phocadownload/GobiernoCorporativo/ guiacolombiana.pdf

Tagiuri, R., \& Davis, J. (1996). Bivalent attributes of the family firm. Family Business Review, 9(2), 199-208. https://doi.org/10.1111/j.17416248.1996.00199.x 